\title{
Persistent exclusion of older people from clinical trials of cardiovascular and antithrombotic medicinal products
}

\author{
Tischa J. M. van der Cammen ${ }^{1}$. Peter Crome ${ }^{2}$
}

Published online: 5 July 2018

(c) European Geriatric Medicine Society 2018

The limited inclusion of older people in clinical trials of medicines intended for use in later life has been extensively discussed for many years. This is of particular importance for conditions such as cardiovascular disease where the prevalence increases with age. Additionally, the inclusion criteria for pivotal clinical trials are formulated so as to create a homogenous population that is not representative of the target population. As a result, age-related variations in disease frequency, the use of concomitant medication, and variations in pharmacokinetic and pharmacodynamic parameters are not adequately reflected in the trial population. The consequences are that the external validity of trial findings is limited with respect to both the benefits and the risks of prescribing any given medication to an older patient.

In the current issue of European Geriatric Medicine, a special article by Cerreta et al., "Medicines for older people. Assessment and transparency at the European Medicines Agency regarding cardiovascular and antithrombotic medicinal products" [1], provides further information on data on this subject. They reviewed the clinical trials for cardiovascular and antithrombotic medicines analysed in support of centralised marketing authorisation by the European Medicines Agency in the period 2006-2016. They report on whether older people were included as participants in the pivotal trials, whether the age distribution of the participants in the registration trials reflected expected use, whether post-authorisation studies were planned, and whether the age distribution of participants was clearly presented in the approval documents.

The results of the analysis of 19 protocols show clearly that older people continue to be excluded from the pivotal

Tischa J. M. van der Cammen

t.j.m.vandercammen@tudelft.nl

1 Faculty of Industrial Design Engineering, Delft University of Technology, Delft, The Netherlands

2 Research Department of Primary Care and Population Health, University College London, London, UK clinical trial protocols, based on a pre-set upper age limit, or are under-represented in the drug registration trials. The proportion of patients over 75 years in antithrombotic registration trials in heart failure and atrial fibrillation does not exceed $20 \%$ and for statins and anti-hypertensives $6 \%$. This is clearly very different from the mean age of the clinical population with cardiovascular pathology. Post-authorisation studies were only planned in $2 / 19$ cases. The authors conclude that, although there is more publicly available information since 2006, data on older patients with frailty and multi-morbidity remain generally scant.

In 2011, the PREDICT study reported agreement among 521 general practitioners, geriatricians, clinical researchers, ethicists, nurses and industry pharmacologists/pharmacists in nine EU countries, that exclusion from clinical trials on age grounds alone was unjustified and that under-representation of older people in trials caused real difficulties for prescribers and patients [2]. In addition, the PREDICT group reported persistent and widespread exclusion of older patients from ongoing clinical trials regarding heart failure [3]. A more recent study reported that despite the high burden of ischaemic heart disease in older adults, the majority of drug trials do not enroll participants reflective of agerelated prevalence of the disease [4].

The European Medicines Agency has recognised that older people are a special group and in 2011, their geriatric medicines strategy was launched $[5,6]$.

The first aim of the strategy, deriving from the awareness that older people are the main users of medications, and not a minority or special population, is to ensure that the use of newly approved medicines in the intended population is supported by relevant data on the benefit-risk balance. The second aim is to improve the availability of information to patients and prescribers, to support safer use of medications. These aims clearly require that medicines must be evaluated in older people.

Despite the increased awareness of this undesirable situation and the resulting initiatives, there is a persistent 
mismatch between the ages of patients included in clinical trials and those treated in clinical practice. Data on older patients with multi-morbidity, frailty and polypharmacy remain scant.

So far, it seems that the implementation of the geriatric medicines strategy has fallen short in substantially changing and improving the situation.

To close the current knowledge gap, the study and evaluation of drugs in all age groups, including older people, needs to be systematically expanded. Licensing authorities should report regularly on the progress of implementing the geriatric medicines strategy for all classes of drugs. External review will continue to be needed.

\section{Compliance with ethical standards}

Conflict of interest The authors declare that they have no conflict of interest.

Ethical approval This article does not contain any studies with human participants or animals performed by any of the authors.

Informed consent For this type of study formal consent is not required.

\section{References}

1. Cerreta F, Padrão A, Skibicka-Stepien I, Strampelli A, de Orbe Izquierdo MS (2018) Medicines for older people: assessment and transparency at the European Medicines Agency regarding cardiovascular and antithrombotic medicinal products. Eur Geriatr Med. https://doi.org/10.1007/s41999-018-0071-1

2. Crome P, Lally F, Cherubini A et al (2011) Exclusion of older people from clinical trials: professional views from nine European countries participating in the PREDICT study. Drugs Aging 28(8):667-677

3. Cherubini A, Oristrell J, Pla X et al (2011) The persistent exclusion of older patients from ongoing clinical trials regarding heart failure. Arch Intern Med 171(6):550-556

4. Bourgeois FT, Orenstein L, Ballakur S, Mandl KD, Ioannidis JP (2017) Exclusion of elderly people from randomized clinical trials of drugs for ischemic heart disease. J Am Geriatr Soc 65(11):2354-2361

5. Cerreta F, Eichler HG, Rasi G (2012) Drug policy for an aging population - the European Medicines Agency's geriatric medicines strategy. N Engl J Med 367:1972-1974

6. EMA Geriatric Medicines Strategy, 17 Feb 2011. http://www.ema. europa.eu/docs/en_GB/document_library/Other/2011/02/WC500 102291.pdf. Accessed 21 Jun 2018 